\section{FRI0376 DIFFERENCES IN RIGHT VENTRICULAR FUNCTION AND MORPHOLOGY IN SSC-PAH AND IPAH ASSESSED BY RIGHT HEART CATHETERIZATION AND CARDIAC MRI}

A.-M. Hoffmann-Vold ${ }^{1}$, E. Hopp ${ }^{2}$, Ø. Midtvedt ${ }^{1}$, T. Garen ${ }^{1}$, P. Szodoray ${ }^{3}$, $\varnothing$. Molberg ${ }^{1}$, A.K. Andreassen ${ }^{4} .{ }^{1}$ Rheumatology; ${ }^{2}$ Radiology; ${ }^{3}$ Immunology;

${ }^{4}$ Cardiology, Oslo University Hospital, Oslo, Norway

Background: Systemic sclerosis (SSc) is a serious disorder with high mortality, mainly driven by cardiopulmonary pathologies including pulmonary arterial hypertension $(P A H)$. Patients with SSc-PAH appear to have worse prognosis than idiopathic PAH (PAH).

Objectives: (A)Compare cardiac magnetic resonance (CMR) of the right ventricle, hemodynamic parameters and immunological markers between patient cohorts with SSc-PAH, iPAH and SSc without PAH, respectively. (B)Evaluate the impact of these variables on $\mathrm{PAH}$-related morbidity and mortality.

Methods: The study cohort included SSc-PAH $(n=16)$, iPAH $(n=15)$ and SSc without PAH $(n=20)$. At baseline, all patients were examined by ventricular volumes by short axis summation of cine CMR, echocardiography and right heart catheterization $(\mathrm{RHC})$. Blood samples were analysed for immune-related factors. At a median follow up of 3 years, clinical data, and new onset of PAH and vital status were registered. A composite outcome for PAH related events was created including: PAH progression, end-stage PAH, hospitalization for PAH worsening and all-cause mortality. Associations between parameters were assessed using ANOVA, Pearson Chi-square, Fishers exact, or independent sample t-test.

Results: Patients with SSc-PAH were significantly older at PAH onset, had lower GMWD, DLCO\% and $\mathrm{Hb}$ than the $\mathrm{PAH}$ patients. RHC results are shown in Table 1. CMR showed that SSc-PAH patients had lower right ventricular mass index (RVMI), RV to left ventricular ratio and RV mass to volume ratio (Table 1). Multiplex immune assays demonstrated higher serum levels of IP-10, VEGF and, IL12 in SSc-PAH than in iPAH. At the end of the study, 12/15 SSc-PAH had developed a PAH-related event. Compared to event-free patients, they were marked by significant CMR changes including higher RVM $(p=0.006)$ and RVMI $(p=0.005)$. In the $\mathrm{PAAH}$ subgroup, $7 / 16$ patients developed an event. These patients were characterized by younger age at onset $(p=0.001)$, higher NT-proBNP $(p=0.017)$, lower 6MWD $(p=0.034)$, higher RVM $(p=0.010)$, RVMI $(p=0.005)$, RV-to-LV ratio $(p=0.001)$ on CMR.

Table 1. Baseline right ventricle hemodynamic and cardiac magnetic resonance measurements

\begin{tabular}{lccc}
\hline & SSc-PAH $(\mathrm{n}=15)$ & iPAH $(\mathrm{n}=16)$ & $\mathrm{p}$-value \\
\hline RHC & & & \\
Cardiac output & $6.0(2.9)$ & $5.4(1.4)$ & n.s. \\
Cardiac index & $3.2(1.5)$ & $2.9(0.6)$ & n.s. \\
RAP, mmHg & $4.6(3.1)$ & $7.2(3.4)$ & 0.007 \\
SPAP, mmHg & $60(19.8)$ & $88(23.3)$ & $<0.001$ \\
mPAP, mmHg & $35.5(11.7)$ & $55(17.1)$ & $<0.001$ \\
PCV, mmHg & $6.2(2.9)$ & $7.4(3.1)$ & n.s. \\
PA oxygen saturation\% & $81.3(33.1)$ & $93.2(31)$ & n.s. \\
PVR (Wood) & $5.4(3.9)$ & $9.7(4.3)$ & $<0.001$ \\
RV MRI & & & \\
RVSV index, ml/m ${ }^{2}$ & $40.9(7.8)$ & $40.4(7.8)$ & n.s. \\
RVESV, ml & $144.9(80.3)$ & $146.8(46.4)$ & n.s. \\
RVEDV, ml & $219.5(84.2)$ & $223.8(52.8)$ & n.s. \\
RVEDV index, ml/m ${ }^{2}$ & $120.8(50.5)$ & $120.8(28.3)$ & n.s. \\
RVEF, \% & $37.1(10.5)$ & $34.5(10.5)$ & n.s. \\
RV mass, g & $50.1(21.3)$ & $72.9(27.4)$ & $<0.001$ \\
RV mass index, g/m ${ }^{2}$ & $26.2(10.8)$ & $38.2(14.9)$ & $<0.001$ \\
RV-to-LV ratio & $0.51(0.14)$ & $0.82(0.32)$ & $<0.001$ \\
RV mass-to-volume ratio, $\mathrm{g} / \mathrm{ml}$ & $0.22(0.06)$ & $0.31(0.08)$ & $<0.001$ \\
\hline
\end{tabular}

Conclusions: The CMR measures indicate that the RV adapts differently in IPAH and SSc-PAH; with more pronounced RV hypertrophy in iPAH patients. The mechanisms behind the differences in RV hypertrophy in SSc-PAH and PAH are not known, but may involve microvascular changes and deregulated inflammatory cascades. This study indicates that CMR may predict PAH progression in SSc. Disclosure of Interest: None declared

DOI: 10.1136/annrheumdis-2017-eular.5372

\section{FRI0377 ABNORMAL NAILFOLD CAPILLAROSCOPY PATTERN IS COMMON IN PATIENTS WITH CONNECTIVE TISSUE DISEASE AND ASSOCIATED WITH ORGAN INVOLVEMENT}

A.M. Van Roon ${ }^{1}$, C.C. Huisman ${ }^{1}$, A.M. van Roon ${ }^{1}$, D. Zhang ${ }^{2}$, A.J. Stel ${ }^{3}$, A.J. Smit ${ }^{1}$, H. Bootsma ${ }^{3}$, D.J. Mulder ${ }^{1} .{ }^{1}$ Internal Medicine - Vascular Medicine, University of Groningen - University Medical Center Groningen, Groningen; ${ }^{2}$ Rheumatology, Medical Center Leeuwarden, Leeuwarden; ${ }^{3}$ Rheumatology and Clinical Immunology, University of Groningen - University Medical Center Groningen, Groningen, Netherlands

Background: Nailfold capillary microscopy (NCM) has been shown to be associated with disease severity and internal organ involvement in non-consecutive systemic sclerosis (SSc) cohorts. NCM may help in early recognition of connective tissue disease (CTD) but it is unclear to which extent NCM abnormalities occur in several CTD and whether these are associated with organ involvement.

Objectives: To assess NCM in consecutive patients with Raynaud's phenomenon (RP) and whether these are associated with signs of organ involvement.
Methods: NCM was assessed in consecutive patients with RP ( $n=759)$ by widefield videocapillaroscopy, according to standardized procedures at a dedicated vascular laboratory by trained and experienced technicians. The pattern was classified as normal $(n=325)$, non-specific $(n=188)$ or SSc pattern (early $(n=106)$, active $(n=140)$ or late $(n=0)$ based on the Cutolo patterns). Potential pulmonary involvement was defined as forced vital capacity or diffusion capacity $<70 \%$. Gastro-intestinal involvement as scintigraphically (Tc-99M colloid) oesophagus dysmotility. Skin involvement as puffy fingers or sclerodactyly.

Patients were classified as primary RP (normal NCM and negative serology, $\mathrm{n}=226$ ) or secondary RP (abnormal NCM and/or serology) groups: early SSc $(n=195)$, SSc $(n=40)$, incomplete Sjögren's syndrome (iSS, $n=5)$, primary SS (pSS, $n=30$ ), incomplete systemic lupus erythematodes (iSLE, $n=42$ ), SLE $(n=30)$, mixed CTD (MCTD, $n=7$ ), rheumatoid arthritis (RA, $n=15$ ) or when not meeting criteria as other $(n=169)$. None were diagnosed with polymyositis or dermatomyositis.

Results: SSc pattern was observed in $33 \%$ of patients with pSS, $17 \%$ SLE, $71 \%$ MCTD and $13 \%$ RA. Pulmonary, oesophageal and skin involvement was more frequent in secondary RP patients with an SSc pattern, even when analysing only those fulfilling definite criteria for CTD or after exclusion of early SSc and SSc patients. For secondary RP patients, absence of an SSc NCM pattern had a high negative predictive value for pulmonary (92\%), oesophageal (91\%) and skin (96\%) involvement, while positive predictive values were low.

Table 1. Nailfold capillaroscopic pattern per patient diagnosis group in patients with Raynaud's phenomenon

\begin{tabular}{|c|c|c|c|c|c|c|c|c|c|c|}
\hline \multirow[t]{3}{*}{ NCM pattern } & \multirow{3}{*}{$\begin{array}{l}\text { Primary } \\
\text { Raynaud }\end{array}$} & \multicolumn{9}{|c|}{ Secondary Raynaud } \\
\hline & & \multicolumn{5}{|c|}{ Definite diagnosis } & \multicolumn{4}{|c|}{ No classifiable disease } \\
\hline & & $\begin{array}{l}\mathrm{SSc} \\
\mathrm{n}=40\end{array}$ & $\begin{array}{l}\mathrm{pSS} \\
\mathrm{n}=30\end{array}$ & $\begin{array}{c}\text { SLE } \\
n=30\end{array}$ & $\begin{array}{c}\text { MCTD } \\
n=7\end{array}$ & $\begin{array}{c}\mathrm{RA} \\
\mathrm{n}=15\end{array}$ & $\begin{array}{c}\text { early SSc } \\
n=195\end{array}$ & $\begin{array}{l}\text { iSS } \\
\mathrm{n}=5\end{array}$ & $\begin{array}{l}\text { iSLE } \\
n=42\end{array}$ & $\begin{array}{l}\text { Other } \\
\mathrm{n}=169\end{array}$ \\
\hline Normal & $226(100)$ & $1(3)$ & $10(33)$ & $12(40)$ & $2(29)$ & $11(73)$ & $4(2)$ & $4(80)$ & $29(69)$ & $26(15)$ \\
\hline Non-specific & $0(0)$ & $4(10)$ & $10(33)$ & $13(43)$ & $0(0)$ & $2(13)$ & $2(1)$ & $1(20)$ & $13(31)$ & $143(85)$ \\
\hline Early & $0(0)$ & $7(18)$ & $4(13)$ & $2(7)$ & $1(14)$ & $1(7)$ & $91(47)$ & $0(0)$ & $0(0)$ & $0(0)$ \\
\hline Active & $0(0)$ & $28(70)$ & $6(20)$ & $3(10)$ & $4(57)$ & $1(7)$ & $98(50)$ & $0(0)$ & $0(0)$ & $0(0)$ \\
\hline
\end{tabular}

Data in number (percentage).

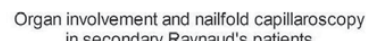

in secondary Raynaud's capillaros

Pulmonary Oesophageal Skin

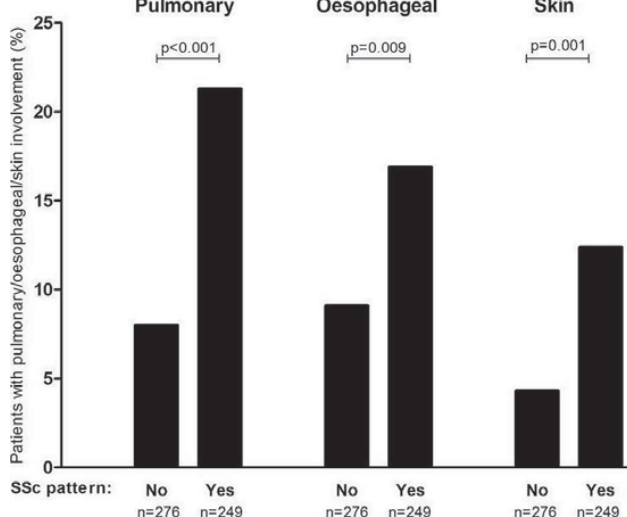

Conclusions: SSc pattern on NCM is common in CTD patients and is associated with frequent prevalence of organ involvement, even in the absence of (early) SSc Although this was a retrospective cohort, these data underline the importance of assessing NCM in RP patients to evaluate the risk for organ involvement in CTD other than SSc, already in early disease stages.

Disclosure of Interest: None declared

DOI: 10.1136/annrheumdis-2017-eular.1390

\section{FRI0378 CARDIAC INVOLVEMENT IN UNDIFFERENTIATED CONNECTIVE TISSUE DISEASE AT RISK FOR SYSTEMIC SCLEROSIS. A TISSUE DOPPLER IMAGING STUDY}

A. Riccardi ${ }^{1}$, M. D'Alto ${ }^{2}$, P. Argiento ${ }^{2}$, E. Romeo ${ }^{2}$, I. Di Stefano ${ }^{1}$, S. Fasano ${ }^{1}$ A. Mattera lacono ${ }^{2}$, A. D'Andrea ${ }^{2}$, A. Sanduzzi ${ }^{3}$, M. Bocchino ${ }^{3}$, L. Docimo ${ }^{4}$, S. Tolone ${ }^{4}$, M.G. Russo ${ }^{2}$, G. Valentini ${ }^{1} .{ }^{1}$ Rheumatology Unit; ${ }^{2}$ Department of Cardiology, University of Campania "Luigi Vanvitelli"; ${ }^{3}$ Department of Clinical Medicine and Surgery, University "Federico II"; ${ }^{4}$ Division of General and Bariatric Surgery, University of Campania "Luigi Vanvitelli", Naples, Italy

Background: Undifferentiated connective tissue disease at risk for Systemic Sclerosis (UCTD-risk-SSc), previously referred to as very early SSc, is a condition characterized by Raynaud's phenomenon (RP) with SSc marker autoantibodies and/or typical capillaroscopic findings and unsatisfying classification criteria for the disease (1). UCTD-risk- SSc patients have been reported to present, in about $40 \%$ of the cases, a preclinical vascular internal organ disease as detected by a diffusing lung capacity for $\mathrm{CO}<80 \%$ of the predicted value (lung involvement) and/or a low esophageal sphincter pressure $<15 \mathrm{mmHg}$ (oesophageal involvement) and/or a mitral $\mathrm{E} / \mathrm{A}$ ratio $<1$ (heart involvement) $(2,3)$. 